\title{
Incerteza em resultados analíticos e verificação de conformidade de qualidade de alimentos
}

\author{
Uncertainty in analytical measurements and compliance assessment of food quality
}

\author{
Elisabeth Borges GONÇALVES ${ }^{1 *}$, Rosemar ANTONIASSI ${ }^{1}$
}

\begin{abstract}
Resumo
Este artigo questiona a validade de estimativas de incerteza (dispersão, variância e seus correlatos, como precisão, desvio padrão, etc.) de medição e incerteza de amostragem, bem como sua validade em verificações de conformidade. Obteve tendências de acréscimo ou comportamento em leque em micotoxinas, sódio, óleos graxos e curvas de calibração para carotenoides em ensaios de proficiência, experimentos de precisão de instituições consagradas ou acreditadas e curvas de calibração, o que invalida diversas estimativas de incerteza de medição. Questionou e recalculou resultados do Guia de Incerteza de Amostragem, demonstrando inadequações teóricas e concluiu por sua invalidade. Propôs sistema de estimação voltada para o alimento/processo que se pesquisa através de plano amostral normalizado ou especificamente elaborado para fim de verificação de conformidade. Finalmente, traz as regras decisórias estocásticas para verificação de conformidade em alimentos e diversos outros produtos.
\end{abstract}

Palavras-chave: variância; validação de métodos analíticos; técnicas de amostragem; controle estatístico de qualidade; controle estatístico de processos (capabilidade); cálculo de incerteza.

\begin{abstract}
This paper discusses the validity of estimators of uncertainty (dispersion, variance and its functions such as precision, standard deviation, and others) of measurements to be used to assess compliance. Estimates of uncertainty in analytical methods measurement were shown describing inconsistent trends in mycotoxins, sodium, grasses oils, and calibration curves for carotenoids mycotoxins. The data were certified by proficiency tests, precision experiments (collaborative studies), and calibration routines. The uncertainty of sampling, was considered, and its invalidity can be concluded. Considering the system of generation of uncertainty as a whole, a systematic estimation for foods and foods processing using standard or specific sampling plans is suggested. Moreover, stochastic rules for verifying compliance in foods and other products were established.

Keywords: variance; validation of analytical methods; sampling techniques; statistical quality control; capability; calculation of uncertainty.
\end{abstract}

\section{Introdução}

\subsection{Movimento da qualidade e definições anteriores}

No movimento atual de qualidade para laboratórios de análise envolvendo diversas instituições nacionais e internacionais, incerteza é, de longe, o requisito de validação de métodos analíticos mais publicado e menos profundamente discutido. Na realidade, trata-se de uma propriedade natural de qualquer medição, mas de um conceito de difícil articulação que requer atenção e cuidado.

Os laboratórios de ensaios devem ter e aplicar procedimentos para cálculo das incertezas de medição (ASSOCIAÇÃO...; INTERNATIONAL ORGANIZATION....; INTERNATIONAL ELETROTECHNICAL..., 2001; INTERNATIONAL ORGANIZATION...; INTERNATIONAL ELETROTECHNICAL..., 2005). Sem o conhecimento quantitativo da "incerteza de medição" seria impossível concluir se diferenças observadas, por exemplo, entre resultados de laboratórios diferentes poderiam ser atribuídas a diferenças de precisão ou outras causas.

Assim, a metrologia é clara ao admitir que pretende usar a incerteza calculada para obter conclusões que, sem dúvida, irão subsidiar uma tomada de decisões.
Segundo a National Association of Testing Authorities (NATA) (1998), a "incerteza de medição" é parte de uma expressão que define um intervalo de valores dentro do qual está o "verdadeiro valor", ou o valor apropriado aceito de um mensurando.

“Incerteza de medição" é, no VIM (Vocabulário Internacional de Metrologia) ou ISO, 1995, conhecido como ISO GUM, ou Instituto Nacional de Metrologia, Normalização e Qualidade Industrial, Confederação Nacional da Indústria, Serviço Nacional de Aprendizagem Industrial, (INMETRO/ CNI/SENAI) (2000), um parâmetro associado a um resultado de medição, que pode ser atribuído a um mensurando que caracteriza a dispersão dos valores. Pode ser um desvio padrão ou múltiplo dele ou, ainda, semiparte do intervalo com certo grau de confiança. No DOQ-CGCRE-008, o Instituto Nacional de Metrologia, Normalização e Qualidade Industrial (INMETRO) (2003), mantém esta definição, uma das raras vezes em que se encontra precisão como sendo incerteza.

Ellison, Roesslein e Williams (2000), no Guia EURACHEM/ CITAC de incerteza de medição, adotam a definição do ISO

${ }^{1}$ Embrapa Agroindústria de Alimentos, Av. das Américas, n. 29.501, Guaratiba, CEP 23020-470, Rio de Janeiro - RJ, Brasil, E-mail: goncaleb@ctaa.embrapa.br

${ }^{*}$ A quem a correspondência deve ser enviada 
GUM. Admitem que o parâmetro abrange muitos componentes e que pode ser, por exemplo, um desvio padrão. O cálculo por componentes exposto nesse Guia e usado generalizadamente especifica o mensurando, identifica as fontes de "incerteza de medição", estima seus correspondentes desvios padrão e traz o cálculo da "incerteza combinada".

A incerteza de medição nesse Guia é expressa por (Equação 1):

$$
u\left(y\left(x_{1}, x_{2}, \ldots\right)\right)=\sqrt{\sum_{i=1}^{n} c_{i}^{2} u\left(x_{i}\right)^{2}}=\sqrt{\sum_{i=1}^{n} u\left(y, x_{i}\right)^{2}}
$$

onde $y\left(x_{1}, x_{2}, \ldots\right)$ é uma função de $x_{1}, x_{2}, \ldots$ e $c_{i}$ é um coeficiente de sensibilidade fornecido por $c_{i}=\delta \mathrm{y} / \delta \mathrm{x}_{\mathrm{i}}$, o diferencial parcial de $\mathrm{y}$ com relação a $x_{i}$. O mesmo tipo de cálculo pode ser encontrado na EA-4/02 (EUROPEAN..., 1999), Taylor e Kuyatt (1993) e UKAS (UNITED..., 2000).

\subsection{Exprimindo "incerteza"}

De acordo com o Inmetro, nos documentos DOQ-CGCRE 008 (INSTITUTO..., 2003, 2007), precisão é um termo geral para avaliar dispersão de resultados entre ensaios independentes repetidos de uma mesma amostra, amostras semelhantes ou padrões em condições definidas, e é usualmente expressa pelo desvio padrão. As duas formas mais comuns de expressá-la seriam a repetitividade e a reprodutibilidade, ambas geralmente dependentes da concentração do analito.

Scheffé (1973) é um clássico conhecido da calibração. Estrutura resultados de medições definindo acurácia através de um modelo constituído de precisão e vício, que é exatamente o que se entrevê em todos os documentos de validação intralaboratorial, inclusive nos DOQ-CGCRE 008 (INSTITUTO..., 2003, 2007).

Estocasticamente, variância (quadrado do desvio padrão), desvio padrão, amplitude, amplitude interquartílica, etc. são todos estimadores de dispersão. A incerteza de medição, comumente notada como $\mu_{x}^{2}$, é exatamente uma variância, razão de ser de toda a Estatística, e a incerteza combinada é a variância de uma função de variáveis aleatórias, como encontrado em qualquer texto da área de estatística matemática e probabilidades, por exemplo, em Hogg e Craig (1978).

Uma vez que incerteza évariância, então é variação, é dispersão; logo "incerteza" é precisão, como admitido e já mencionado em DOQ-CGCRE 008 (INSTITUTO..., 2003, 2007), anteriormente mencionado. Assim, deste ponto em diante, "incerteza" será usada também como precisão. De modo a abarcar um público mais amplo, este artigo passará a se referir à mesma propriedade usando três termos: variância, incerteza ou precisão.

Finalmente, é a estatística que "permite avaliar e estudar as incertezas e os seus efeitos no planejamento e interpretação de experiências" (MATSUSHITA, 2008). Então, continuarão a ser explorados conceitos e aplicações pertinentes da literatura em qualidade para laboratórios, para depois, usando resultados de ensaios ou experimentos de alto grau de confiabilidade, rediscuti-los estocasticamente.

\subsection{Um outro efeito que influenciaria o resultado de medição}

A avaliação de incerteza de medição através da decomposição em seus componentes, exposta no guia mencionado e outros, tem recebido várias críticas. Como exemplo, ela se aplica muito pouco na ausência de um modelo (estocástico, genérico) compreensível do processo de medição, Working Party on Chemical Contaminants in Foods (WPCC) (2003).

Horwitz e Albert (INTERNATIONAL..., 1997) comentam que os cálculos de incerteza recomendados para medidas físicas não são transferidos imediatamente para a química porque estas medidas têm componentes de erro e comportamento de repetições diferentes. Nos sistemas químicos, os erros aleatórios predominam e vícios são difíceis de identificar e remover. Assim, vícios devem ser controlados no laboratório.

A ISO/TS 21748 (2004) apresenta a avaliação de incerteza de medição (precisão de medição ou variação de medição ou correlatos), usando dados obtidos de experimentos de precisão (estudos colaborativos). Prova que as abordagens de cálculo de incerteza de medição por componentes e de cálculo baseado nestes experimentos são equivalentes e que ambas estão de acordo com a definição de incerteza do ISO GUM. Então, admite a reprodutibilidade como estimativa de incerteza de medição.

Complementarmente, ambas, "repetitividade e reprodutibilidade, são geralmente dependentes da concentração do analito" e "devem ser determinadas para um diferente número de concentrações" (INSTITUTO..., 2003, 2007). Estes documentos consideram que, em "casos relevantes, a relação entre a precisão e a concentração do analito deve ser estabelecida".

Foi recentemente publicada em Ramsey e Ellison (2007) a incerteza proveniente de "amostragem", no guia conhecido por Guia de incerteza de amostragem de EURACHEM/EUROLAB/ CITAC/Nordtest/AMC. Nele se inclui a amostragem como outra fonte de "incerteza de medição" e se considera que o principal objetivo da medição é possibilitar a tomada de decisões.

Esse Guia admite as "incertezas", a de medição e a de "amostragem", e demonstra a necessidade de se dispor de estimativas de incerteza de "todas as partes do processo de medição", incluindo a de "amostragem e de preparação de amostras".

Segundo esse Guia, a incerteza incluiria todos os efeitos (erros sistemáticos e aleatórios) que poderiam influenciar um resultado, e a diferença entre homogeneidade e heterogeneidade, ou seja, como o grau em que uma propriedade ou constituinte é distribuída em uma quantidade de material. A heterogeneidade do material estaria entre os fatores mais importantes que contribuiriam para a incerteza de amostragem.

Admite estimativas de incerteza obtidas a partir de componentes de variância de modelos de delineamento experimental aplicados em coleta de dados. Em seu primeiro exemplo, usa coleta sistemática de unidades amostrais (em W) e sugere estabelecer como principais causas de variação no modelo de delineamento "amostras" (locais, canteiros ou áreas de produção), em que coleta 10 pés de alface e reserva 2 pés, e dentro das "amostras" (locais, canteiros ou áreas de produção), assim obtém $2 \times(10-2)$ pés de alface, conforme Figura 1 . 
A variação "entre amostras" seria proveniente das 2 coletas e a variação "dentro das amostras" proviria das duplicatas de análises e significaria "variação analítica”. A “incerteza de medição" seria a soma de ambas.

O Guia generaliza essa abordagem, incluindo outros critérios de classificação no modelo de delineamento. Acrescenta que, por vezes, uma estimativa de vício seja obtida por meio do uso de material de referência, mas, não a usa.

Finalmente, fica claro no Guia EURACHEM/CITAC de conformidade, Ellison e Williams (2007), que o objetivo dos atuais cálculos de incerteza não é meramente de explicação ou de testes de variação, mas de verificação de conformidade, ou seja, utilizar o resultado dos cálculos para decidir se ele indica conformidade ou não com uma especificação. Literalmente menciona que, para isto, "é necessário considerar a incerteza de medição".

Esse Guia de conformidade traz a Figura 2 que usa resultados de cálculos de incerteza e mostra conclusões a serem obtidas perante uma especificação; neste caso, um limite superior a ser obedecido. Apresenta os 4 casos possíveis nestas condições e ressalta que a informação de uma baixa probabilidade de decisão incorreta pode não ser suficiente para se tomar decisões sobre conformidade nos casos (b) e (c).

Por outro lado, a FAO/WHO (FOOD...; WORLD..., 2008) sugere pensar em todo um sistema de medição, ressaltando que há diferença entre a incerteza de medição e conformidade com especificações. Inclui vício (bias) e recuperação como fontes de incerteza, e pergunta se o que se busca é estimar incerteza de medição ou decidir se amostras estão conformes com uma especificação.

Frente ao exposto, o objetivo deste trabalho foi verificar: a) a possibilidade de obtenção de estimativas de incerteza estatisticamente válidas baseado em dados de alto grau de confiança, principalmente ensaios de proficiência conforme (ASSOCIAÇÃO ...; INTERNATIONAL ORGANIZATION...; INTERNATIONAL ELETROTECHNICAL..., 1999), NBR/ISO/ IEC Guia 43-1, emanados até o início de 2008, experimentos de precisão, publicações citadas em normas; b) refazer cálculos e fazer outros cálculos novos explorando-os; bem como; c) rediscutir a abordagem deste Guia para emprego em verificações de conformidade em alimentos.

\section{Material e métodos}

Os analitos estudados foram: a) micotoxinas: aflatoxinas (AFLA) B1 e B2; e ocratoxina A; b) físico-químicos: cinzas, fibra crua, sódio, nitrogênio total; e c) decanal e os ácidos graxos: C 16:0, palmítico, C 18:0, esteárico, e C18:01, oleico.

Os dados usados de médias e incertezas desses analitos foram provenientes: a) de laudos de ensaios de proficiência ou de experimentos de precisão de instituições consagradas ou acreditadas; ou b) de exemplos dos próprios Guias referenciados neste artigo; ou ainda; c) de curva de calibração ou experimento com uso de fortificação em níveis conhecidos. Portanto, trata-se, na maior parte, de resultados de alto grau de confiabilidade nos dois primeiros casos, enquanto que de experimentos simples no último caso.
Os materiais empregados foram resultados de laudos emitidos pelos provedores: Food Analysis and Performance Assessment Scheme (FAPAS), no período 2001 a 2003; Fundação de Ciência e Tecnologia (CIENTEC), de 2001 a 2003; American Oil Chemists' Society (AOCS), de 2001 a 2003; e Programa Interlaboratorial de Análise de Trigo e de Farinha de Trigo, da Embrapa Trigo, de 1995 a 2002, dos quais a Embrapa Agroindústria de Alimentos participou.

Os ensaios FAPAS utilizados foram realizados com mais de 50 laboratórios para nitrogênio total e com mais de 10 laboratórios nos ensaios AOCS. Frequentemente mais de 20 laboratórios realizaram análises de farinhas nos ensaios interlaboratoriais da Embrapa Trigo e mais de 10 laboratórios nos ensaios do IPT.

Foram utilizados também resultados dos experimentos de precisão (estudos colaborativos) do programa do Instituto de Pesquisas Tecnológicas, IPT/AAQMR/LAQO de 1994 a 2001, para decanal em óleos essenciais de laranja.

O modelo a um critério de classificação da ISO 5725 -1 (INTERNATIONAL..., 1994a) e ISO 5725 - 2 (1994b) e seus componentes de variância foram a base teórica da interpretação das formas de precisão mais comuns em laboratórios de análises, bem como da variação total implícita neste modelo.

As matrizes para analitos de natureza físico-química foram: cubos de pão torrado; padrão de lisina; cereal matinal; padrão de metionina; carne enlatada; ração suína; refeição com canola; ração para aves; leite condensado; farelo com aveia; refeição com óleo de algodão; concentrado proteico; cereal matinal extrusado; refeição com açafrão; farelo de soja; soja em grão; farinha de carne; refeição de soja; farinha de centeio; leite em pó; refeição com quirera de soja; e farinha de trigo.

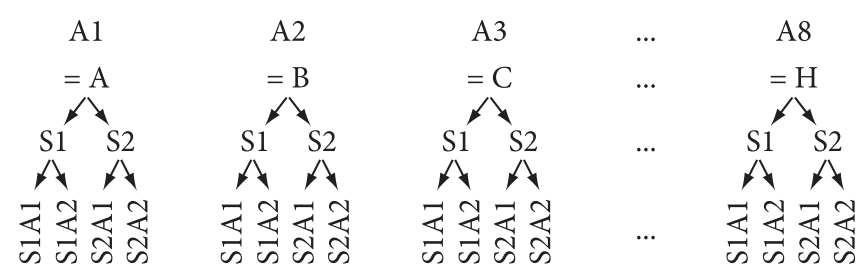

Figura 1. Esquema de nomenclatura das unidades de medição espelhando o primeiro exemplo de Ramsey e Ellison (2007).

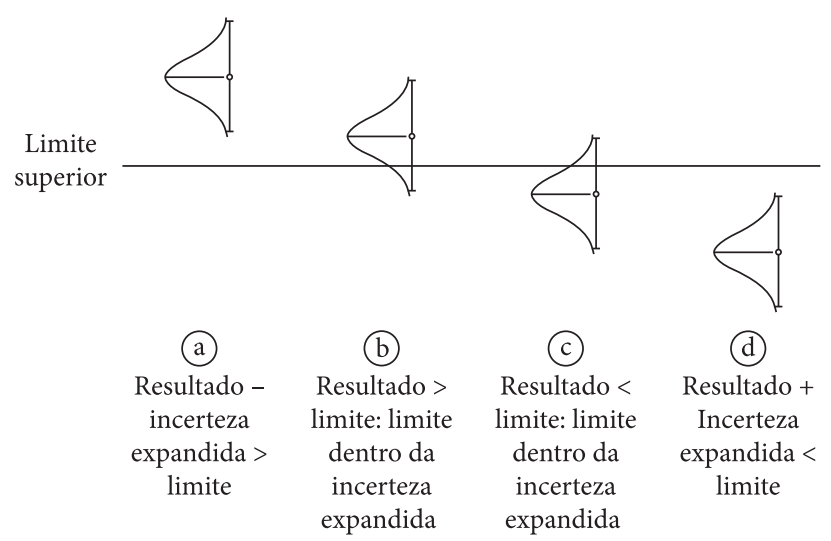

Figura 2. Cenários da verificação de conformidade com limite de especificação do tipo "superior a". Fonte: Ellison e Williams (2007). 
Para aflatoxinas, foram pesquisados cereal matinal de milho, amendoim em pó, frutas secas, pistácio e milho; para ocratoxina A, cafés verdes, cereal e café torrado; enquanto que para óleos essenciais, óleos essenciais de laranja; e, para farinhas, farinha de trigo.

Padrões foram usados na curva de calibração de carotenoides; e café torrado e moído foi adicionado de milho para se medir este contaminante em café em pó.

\section{Resultados e discussão}

Embora o uso de aspas viesse sendo usado para reforçar os conceitos do ponto de vista metrológicos, a partir deste item os termos assim grafados poderão ser discutidos neste artigo frente a fenômenos naturais e conceitos estocásticos.

\subsection{Algumas micotoxinas e medidas físico-químicas}

Na Figura 3, pôde-se observar o comportamento das incertezas certificadas ao longo do eixo do teor de aflatoxinas B1 e B2 (AFLA B1 e AFLA B2) e ocratoxina A (OTA). O que se notou nesses três casos foi, pelo menos aparentemente, tendência crescente ao longo do eixo.

$\mathrm{Na}$ Figura 4, incertezas em função dos teores de cinzas (a), fibra crua (b) e sódio (c), o caso (c) sugeriu tendência crescente. Fato similar ocorreu na Figura 5 para nitrogênio total. A Figura 4a, para cinzas, não demonstrou um comportamento de incerteza muito definido, podendo se tratar de tendência crescente ou comportamento em leque, enquanto que no teor de fibras, Figura 4b, o comportamento em leque parece ser a forma descrita pela respectiva incerteza certificada.
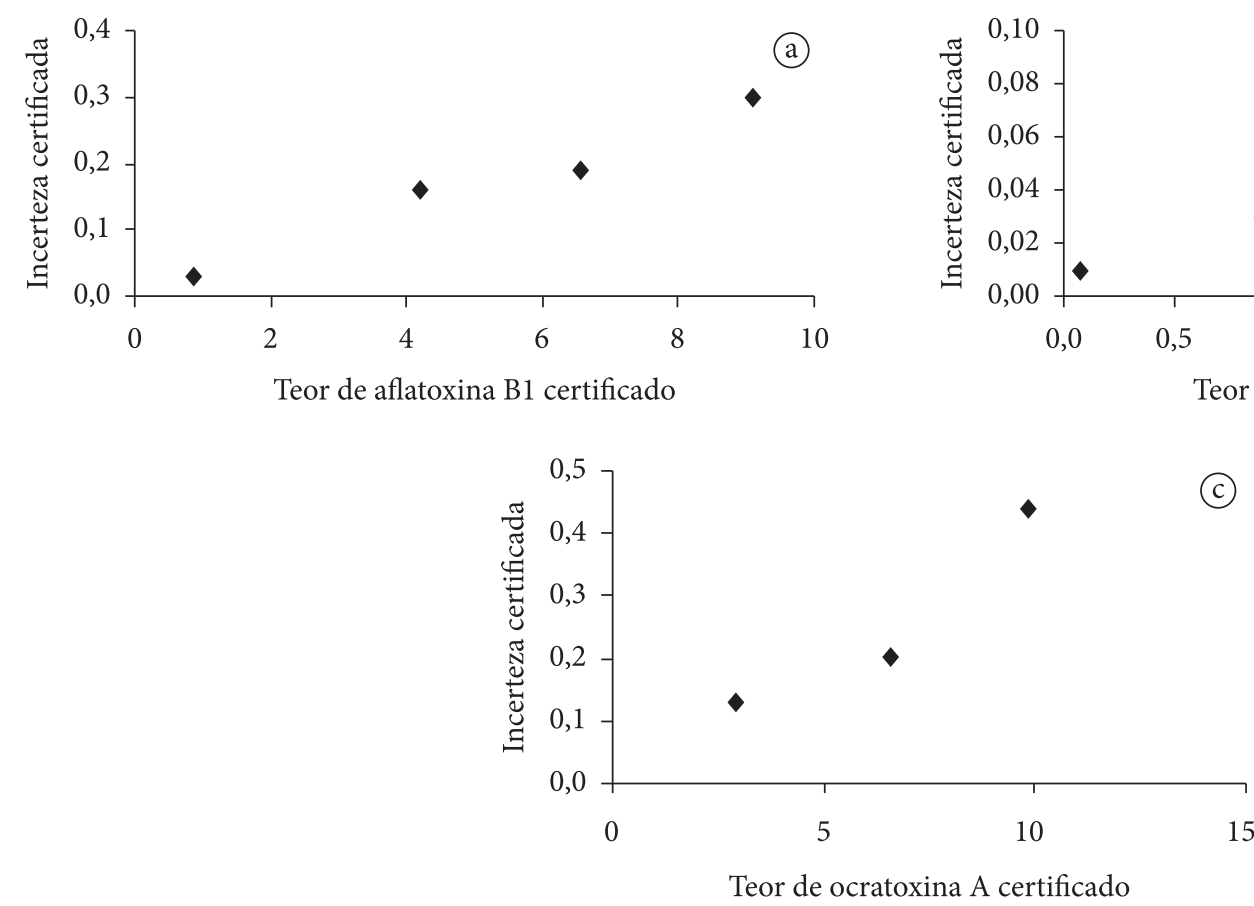

Figura 3. Incertezas certificadas segundo teor certificado da micotoxina estudada em ensaios de proficiência FAPAS - a) aflatoxina B1; b) aflatoxina B2; e c) ocratoxina A.
Nos casos da Figura 3 e Figura 4c, a relação analito $x$ “incerteza” seria, a princípio, linear. Na Figura 5, para nitrogênio total $(\mathrm{N})$ com mais detalhe, entre 3\% e aproximadamente $8 \%$ encontrou-se uma região com tendência estacionária, comportamento esperado na faixa de trabalho de um método. No entanto, no intervalo inferior a 3 os desvios padrão tenderam a se apresentar em níveis inferiores aos da faixa de trabalho, enquanto que ocorreu o contrário na região superior a pouco mais de $8 \%$.

O que se depreendeu desses resultados foi que a faixa de trabalho para $\mathrm{N}$ total seria exatamente aquela em que o comportamento do desvio padrão parece aproximadamente estacionário. Mas, isto não significa que não podem ocorrer diferenças significativas entre desvios padrão (incertezas padrão) nesta faixa, como apresentados em Gonçalves et al. (2006).

\subsection{Repetitividade e reprodutibilidade em decanal}

Houve oscilações significativas tanto na repetitividade quanto na reprodutibilidade nos experimentos de precisão (estudos colaborativos) para este analito em óleos de laranja, Figura 6. Ainda, mesmo em níveis decanal muito próximos, como entre 0,1782 e 0,1809, foram encontradas variações significativas em Gonçalves et al. (2006). Isto se observou na variação total, na repetitividade e na reprodutibilidade.

Pôde-se notar tendência crescente na reprodutibilidade no decorrer do eixo do analito. De modo similar, ao se aplicar zoom para a repetitividade na Figura $6 b$, a tendência de acréscimo ao longo do eixo do analito é notória, ainda que com coeficiente de crescimento mais baixo que o obtido para a reprodutibilidade.

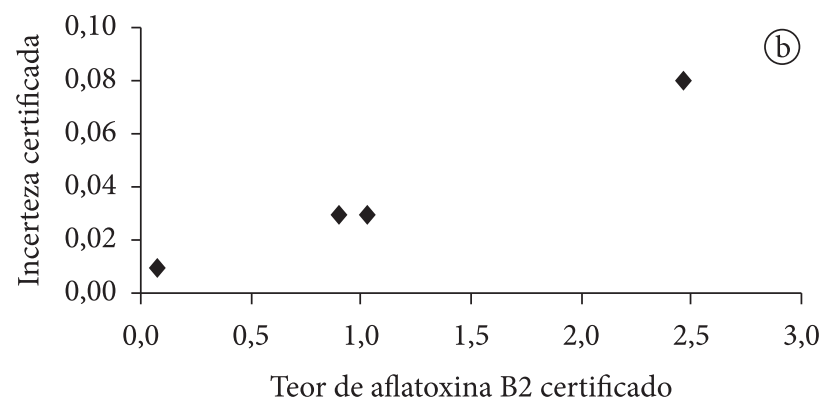

(c)
Teor de ocratoxina A certificado 
Assim, embora a Figura 6a parecesse, a princípio, demonstrar pequena variação (na vertical) para a repetitividade, ambas as formas de precisão demonstraram tendência de acréscimo ao longo do eixo do decanal (eixo x).

\section{3 Ácidos graxos}

Na Figura 7, de ensaios AOCS para alguns ácidos graxos bastante comuns, o acréscimo dos desvios padrão ao longo dos eixos das concentrações dos analitos também foi notado, embora ainda não reforçado pela pequena quantidade disponível de dados.

Assim, a existência de uma tendência crescente dos desvios padrão (precisões, incertezas padrão) ao longo dos eixos dos analitos foi apresentada por vários casos de grande importância na caracterização e qualidade de alimentos, podendo estar presente, inclusive, em óleos graxos.

Embora este tipo de tendência não chegue a invalidar o método em que ocorre, como mencionam Thompson, Stephen e Wood (2002), traz consequências que serão discutidas no decorrer deste artigo.

\subsection{Comportamento em leque}

A Figura $4 b$, de incerteza certificada em função do teor de AFLA B2, claramente exibe comportamento em leque no decorrer do eixo do teor (concentração). Não se logrou nenhum resultado que inibisse este comportamento com uso de transformações cabíveis.
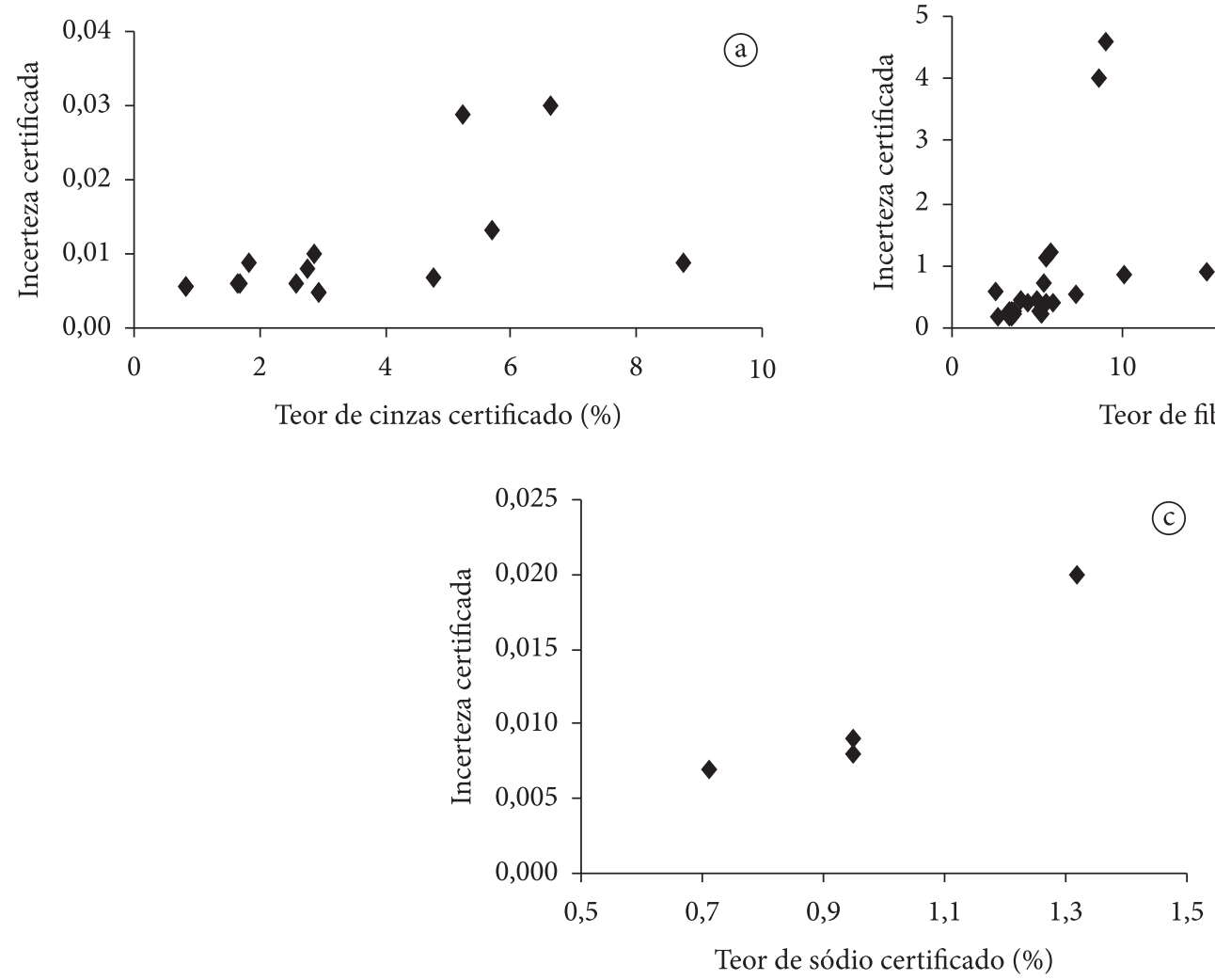

Fato similar ocorreu nas Figuras 8 e 9 de resíduos de linearidade. Na Figura 8 o que se tem é medição de um contaminante que, sem dúvida, encontrará um limite superior, por exemplo, 50\%, ou então o alimento não será aceito como café em pó.

A Figura 9 é de resíduos de ajuste de modelo linear na quantificação do sistema de cromatografia líquida de alta eficiência (HPLC), ou seja, na curva de calibração. Isto é origem de parte da imprecisão nos resultados na medição do analito; neste caso, $\beta$-caroteno. Então, novamente foi obtida formação em leque, preocupante neste caso, pois representa uma estrutura de erros correlacionados que pode não ser esperada em medições em HPLC, e que é um problema para o qual dificilmente se encontra solução adequada.

\subsection{Tendências, formas geométricas e "incertezas"}

Pelo exposto, tendências e formas geométricas indesejáveis foram encontradas nas "incertezas"/imprecisões/variâncias (e seus correspondentes desvios padrão) que, embora não invalidem os métodos analíticos, invalidam estimadores comuns de "incerteza".

Estes casos são, exatamente, do tipo mencionado por DOQCGCRE 008 (INSTITUTO..., 2003, 2007), em que incertezas/ imprecisões/variâncias (e seus correspondentes desvios padrão) são dependentes da concentração do analito.

Por vezes, ficou clara a relação linear positiva entre o teor do analito e a "incerteza" obtida na sua medição. A formação

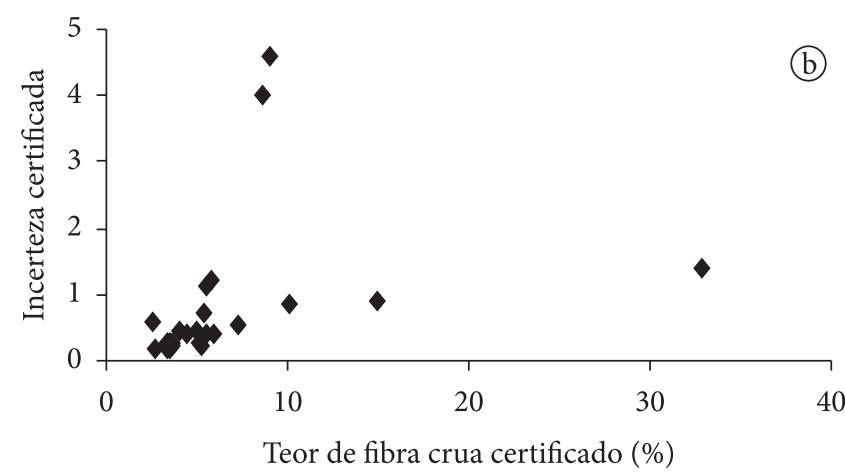

(c) 1,5

Teor de sódio certificado (\%)

Figura 4. Incertezas certificadas em função de teores certificados de medições de a) cinzas; b) fibra crua; e c) sódio em ensaios FAPAS de proficiência. 
em leque observada em outros casos comumente não aceita que se estabeleça uma relação. Além disso, nem mesmo "incerteza" como uma relação em função do teor do analito é prevista em metrologia, pois também contraria a própria definição de "incerteza de medição".

"Incerteza” é um parâmetro associado a uma medição, uma variância ou seu correspondente desvio padrão, definição encontrada em textos de estatística ou no próprio VIM, e não uma função. "Incerteza" (variância) é a dispersão da variável aleatória (mensurando), um número, e tem unidade de medida quadrática, por exemplo, variância de $400 \mathrm{ppb}^{2}$, que implica um desvio padrão de $20 \mathrm{ppb}$.

Em ambos os casos, os DOQ-CGCRE 008 (INSTITUTO..., 2003, 2007) sugerem que precisões (incertezas, variâncias, desvios padrão) sejam "determinadas por diferentes números de concentrações" e que, em "casos relevantes, a relação entre precisão e a concentração do analito deve ser estabelecida”. Isto não encontra fundamento frente às considerações já apresentadas.

Ainda, esses documentos sugerem que o desvio padrão relativo (coeficiente de variação) "pode ser mais útil, pois foi normalizado com base na concentração e é praticamente

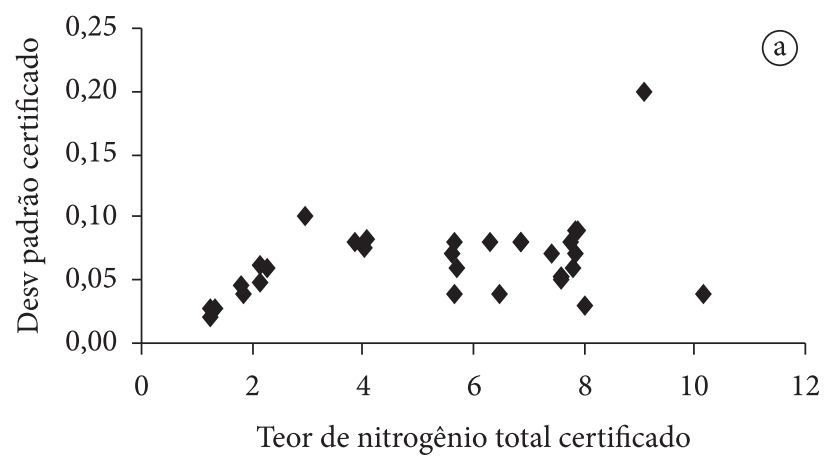

constante na faixa de interesse, contanto que esta não seja muito grande", porém, comparar com os coeficientes de variação quando os teores dos analitos (médias) variam, não tem justificativa técnica. E ainda, se os desvios padrão apresentam acréscimo ao longo do eixo do analito (eixo x), o coeficiente de variação somente seria constante se o coeficiente da relação linear fosse 1 .

Este tipo de problema pode se reduzir se for restringida a faixa de trabalho do método, ou fixada uma região de interesse.

Continuando os comentários sobre as propostas nesses documentos, determinar a variância em diferentes números de concentrações na rotina de laboratórios de serviços de análises não é razoável porque existe uma relação linear que não seria resolvida desta forma.

E, que resultado prático poderia ter tentar estabelecer essa relação se já foi demonstrado que há variações significativas nas variâncias em concentrações muito próximas dos analitos e também em matrizes de mesma natureza? E qual seria a utilidade de usar diferentes concentrações em um serviço de análise em que as matrizes se sucedem sem previsão de qual

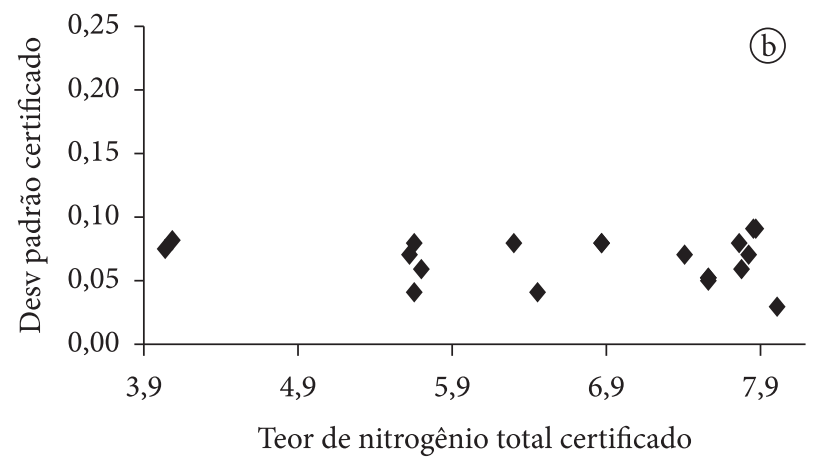

Figura 5. Desvios padrão certificados em função de teores certificados de nitrogênio total em ensaios FAPAS, CIENTEC e AOCS de proficiência apresentados em 2 intervalos (a e b) do eixo de nitrogênio total (\%).
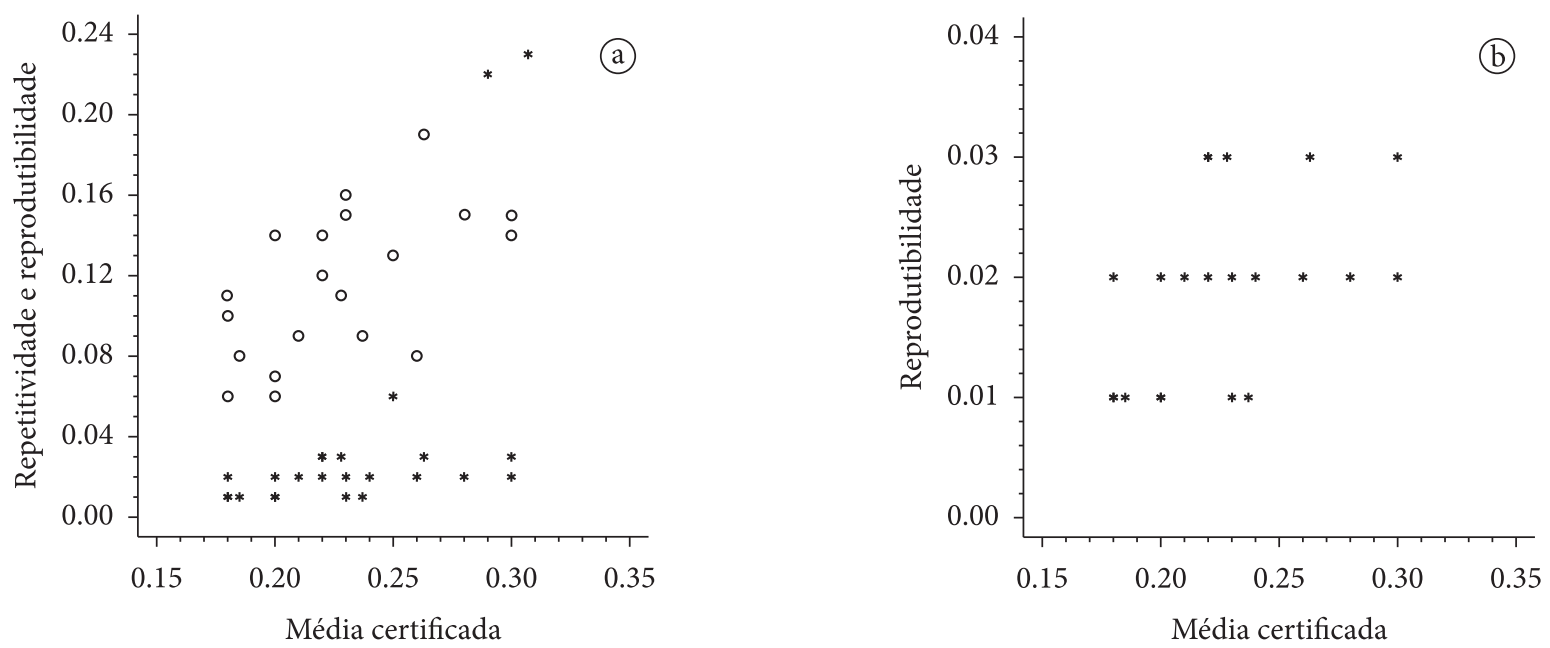

$$
\text { - Repetitividade * Reprodutibilidade }
$$

Figura 6. a) Repetitividade e reprodutibilidade, e b) repetitividade em zoom segundo média certificada de teor de decanal em óleos essenciais de laranja. 

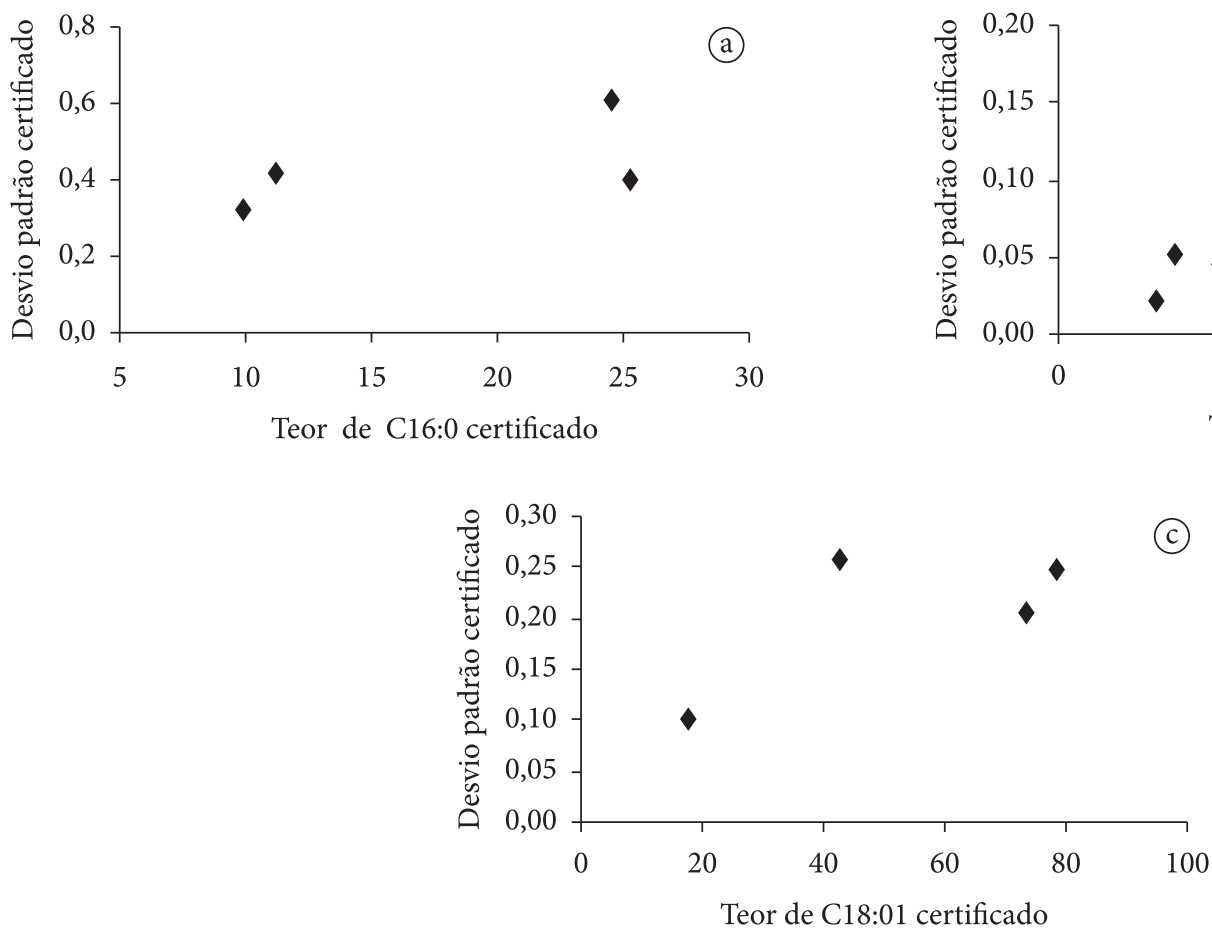

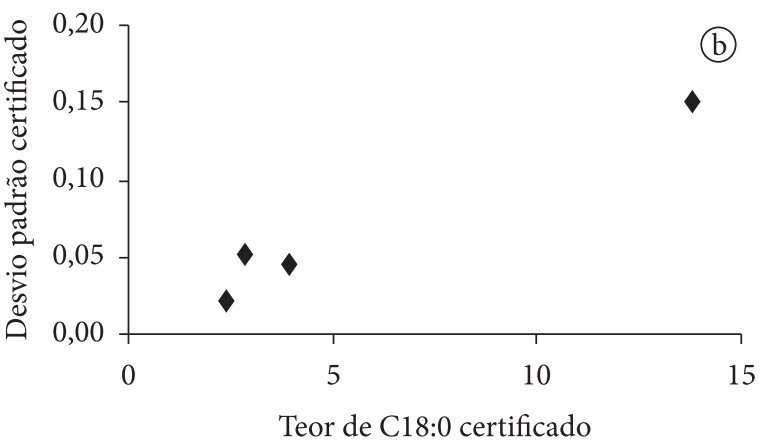

c)

Figura 7. Desvios padrão certificados em função de teores certificados, de alguns ácidos graxos (C 16:0, palmítico; C 18:0, esteárico; e C18:01, oleico).

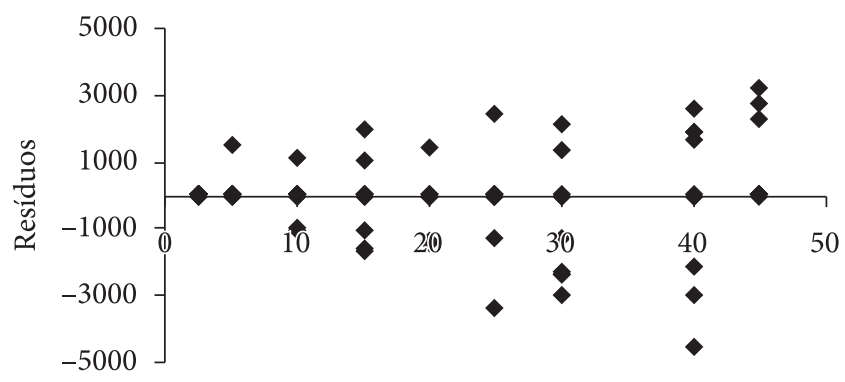

Teor de contaminante $(\%)$

Figura 8. Resíduos do ajuste na verificação de linearidade em café em pó segundo teor (\%) do contaminante (milho).

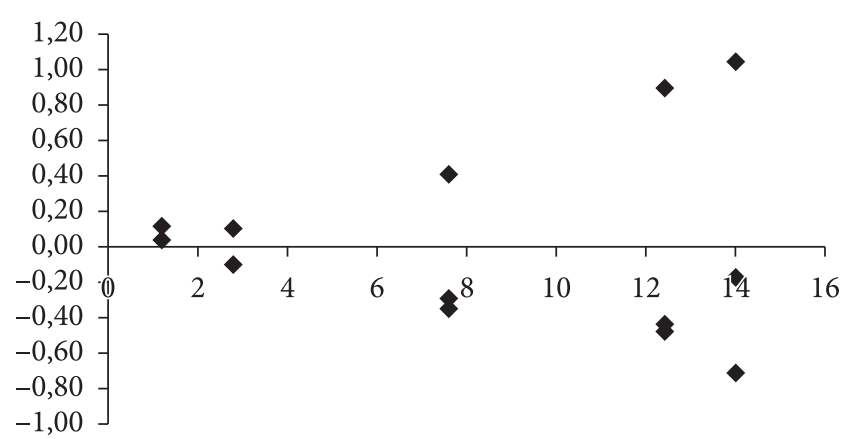

Teor de padrão

Figura 9. Resíduos do ajuste da curva (reta) de calibração para $\beta$-caroteno. será a próxima ou que nível de analito teriam essas matrizes quando isto é justamente o que está sendo medido? Finalmente, nos casos de variância (incerteza) com componente de formação em leque, nenhuma destas duas sugestões seria válida.

Ou seja, laboratórios de serviços de análises, na esmagadora maioria dos casos, não conseguem ter controle efetivo de incerteza para as matrizes que analisam. Como se vê na literatura, até mesmo matrizes com baixos teores de umidade sofrem variações significativas em suas incertezas. Mas, o velho problema de manutenção de padrões ainda tem reflexos na rotina laboratorial incluindo-se na estimação de incertezas (variâncias), então os laboratórios não têm muitas opções além de tentar manter o controle de incertezas com soluções puras.

\subsection{A base da abordagem da atual "incerteza proveniente da amostragem"}

Como já se demonstrou, a abordagem da incerteza de medição através dos seus componentes (calculando variância de funções de variáveis aleatórias) não é suficiente, e nem mesmo válida para ser usada em diversos analitos em alimentos.

A matriz não é o único fator interferente nos resultados e na sua variância. Há os casos de nível - dependência (dependência da concentração), apresentados de 3.1 a 3.5, e em outros.

Ramsey e Ellison (2007), em seu Guia de "incerteza de medição proveniente de amostragem", como no seu exemplo A1, ao incluir certas "amostras" em seu modelo, teriam demonstrado a contribuição da incerteza da "heterogeneidade" da matriz no cálculo de incerteza de medição. 
Esse Guia usa o mesmo tipo de recurso dos experimentos de precisão (estudos colaborativos), ou seja, emprega o mesmo tipo de estimação de componentes de variância em modelo de delineamento experimental com o artifício de duplicar tomadas de "amostras" e de análises de unidades amostrais para poder ter "amostras" como critério de variação.

Assim, trazem a adaptação de um modelo com dois critérios de classificação ("amostras" ou canteiros e análises dentro das "amostras") como sendo a solução que reuniria a variação associada por esses autores à heterogeneidade das matrizes no fator "amostra".

\subsection{Crítica da incerteza proveniente da amostragem}

\section{Coleta de amostras}

O uso do dito "W", ou seja, zigue-zague nesse Guia na coleta de material é característico da amostragem sistemática, tecnicamente conhecida como um tipo de amostragem dos mais arriscados.

Se o material for colhido nos vértices dessa forma geométrica $(\mathrm{W})$, está sendo colhido próximo às bordaduras $(W$ ou $\bar{W}$ ), poderá não ter a mesma constituição que o do centro dos canteiros. O inverso pode ocorrer se forem usados os centros da forma geométrica (W), ou seja, centros dos canteiros, que poderão ter constituição muito próxima entre si.

Assim, trata-se de um tipo de amostragem que pode subestimar a variância (incerteza, desvio padrão, imprecisão) e deslocar a média do analito (mensurando), especialmente se as bordaduras dos canteiros não realizarem um bom isolamento.

Além disso, embora esses autores mencionem a aleatorização, técnica que possibilita escapar de tendências experimentais e mantém a independência dos resultados, no Exemplo A1 e em outros do Guia de incerteza de amostragem seu uso não foi mencionado. Ainda, foram tomadas 2 porções de cada "amostra" (subunidades ou subamostras) para análise, porém não foi mencionado se foram cegas, independentes, ou qual o plano de medição e codificação usado.

Em função disso, o que se espera é dependência e subestimação da variação entre subunidades, hipóteses em que o modelo de delineamento que o Guia propõe não seria válido.

\section{Modelagem}

Esse Guia é produto de abordagem de incerteza (variância) por modelagem estocástica, uma forma de se tentar quantificar, através de experimentos adequados e modelos estatísticos correspondentes, uma incerteza dita proveniente de "amostragem".

Reestruturando o experimento do exemplo A1, obtevese a Figura 10, em que o experimento é mais detalhado e o delineamento é reconhecido como em classificação hierárquica.

Portanto, contrariando a notação apresentada no Guia, as alfaces, que são, na realidade, 16, ALF1 a ALF16 na Figura 10, não se repetem em cada canteiro $\mathrm{A}$ a $\mathrm{H}$, os quartos são $32 \mathrm{e} o$ modelo de delineamento experimental usado é um modelo de classificação hierárquica, conforme Equação 2:

$y_{i j k}=\mu+c_{j}+a_{i j}+\varepsilon_{i j k}$,

onde $y_{i j k}$ é o valor observado (medido), $\mu$ a média, $c_{j}$ efeito do canteiro $_{\mathrm{i}}, \mathrm{j}=1,2, \ldots, 8, a_{i j}$ a alface $\mathrm{i}_{\mathrm{i} ;}, \mathrm{i}=1,2, \ldots 16$ e $\varepsilon_{i j k}$ o erro de análises, $\mathrm{k}=1,2, \ldots 32 \mathrm{o}$ erro aleatório nos quartos $\mathrm{k}_{\mathrm{k}}$.

Empregando-se esse modelo, foram obtidos estimadores de componentes de variância através da análise de variância decorrente do modelo na Tabela 1.

Em ambos os casos, original e com descarte da dupla de valores aberrantes (outliers) do canteiro 3 ("amostra" 3 ), na Tabela 1, os efeitos de canteiros (entre canteiros ou entre
A1
$=\mathrm{A}$
$\wedge$
ALF1 ALF2

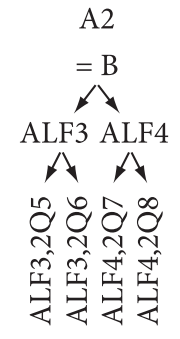
A3
$=\mathrm{C}$

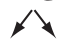
ALF5 ALF6
... $\mathrm{Al}$
... $=\mathrm{H}$
$=\mathrm{H}$
... ALF15 ALF16
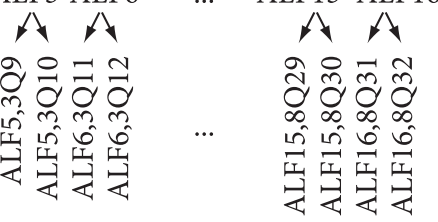

Figura 10. Esquema da realização do exemplo A1 de modo a propiciar visualização do modelo de delineamento experimental em classificação hierárquica. ALFijQk: resultado da análise da alface i no j-ésimo canteiro no quarto k. Nota: exemplo A1 de Ramsey e Ellison (2007) representado em outra estrutura.

Tabela 1. Esquema de análise de variância do modelo adotado no exemplo A1.

\begin{tabular}{clrrrr}
\hline Modelo & \multicolumn{1}{c}{ Causas variação } & GL & SQ & QM & F \\
\hline Exemplo A1 & Entre canteiros & 7 & 12577112,88 & 1796730,41 & $81,83^{*}$ \\
& Alfaces d. canteiros & 8 & 4471511,00 & 558938,87 & $25,46^{*}$ \\
& Análises (quartos, erros aleatórios) & 21 & 351320,00 & 21957,50 \\
& Total & 31 & 17399943,88 & & \\
& Entre canteiros & 7 & 12052056,87 & 1721722,41 & $82,16^{*}$ \\
& Alfaces d. canteiros & 8 & 2278359,50 & 284794,94 & $13,59^{*}$ \\
& Análises (quartos, erros aleatórios) & 14 & 293387,00 & 20956,21 \\
& Total & 29 & 14623803,37 & \\
\hline
\end{tabular}

GL: Graus de Liberdade; SQ: Soma de Quadrados; QM: Quadrado Médio; F: F - Snedecor; *significativo com p < 0,05 d.: dentro; E(QM): Esperanças de Quadrados Médios; Nota: referente ao Guia de incerteza de amostragem de Ramsey e Ellison (2007). 
"amostras"), foi significativo. Assim, os canteiros, ou grupos deles não teriam a mesma média. Fato similar ocorreu com os quartos de alfaces ("subamostras").

Obtendo-se os componentes de variância no caso original, as estimativas resultantes foram: variância de canteiros (ou entre canteiros $)=309447,9$; de alfaces dentro de canteiros $=2684907$; e de erros (análises dos quartos dentro de alfaces) $=29157,5$. Estas variâncias têm seus desvios padrão na Tabela 2, que apresenta também a incerteza de medição considerada em cada caso.

Inicialmente, as propriedades estocásticas do modelo original e com descarte não diferiram para fins de ajuste de modelo (coeficiente de modelagem original obtido $=0,979809$ e com descarte $=0,979938$ ); os erros aleatórios atribuídos aos erros de análises, também não se alteraram muito $(<5 \%)$.

Porém, a incerteza padrão de medição no exemplo A1, usando estimação de máxima verossimilhança $(538,9325)$, foi extremamente alta se comparada às demais $(360,5506 \mathrm{e}$ 369,3168 , correspondendo a F1 = 2,23 * e F2 = 2,13 *; logo essa incerteza padrão foi significativamente maior que as demais). Assim, realmente era exigida uma solução para os valores aberrantes, mas as estimativas obtidas por descarte destes valores e ajuste do mesmo modelo, contudo não balanceado, resultaram em estimativas que não demonstraram justificar o emprego de estimadores robustos.

Diferenças de análises ocorreram normalmente, especialmente em porções diferentes (quartos dentro de alfaces), isto é completamente natural e faz parte da variação total nos resultados.

\section{Modelagem versus amostragem}

Incluir canteiros ("amostras") como causa de variação (ou fonte de variação ou critério de classificação), como no Guia de incerteza de amostragem, e obtê-los ou admiti-los como significativos, contraria o conceito estocástico de amostra.

Uma amostra no seu sentido estocástico tem seus elementos (canteiros) independentes e identicamente distribuídas. Portanto, médias de canteiros significativamente diferentes na realização do teste F-Snedecor, como consta na Tabela 1, contrariam o que se espera da amostragem. Logo, o efeito de canteiros não deveria ter sido significativo.

Frente à realidade que se espera de amostras estocasticamente planejadas e coletadas, o modelo proposto pelo Guia deveria ser reestruturado, de maneira que o critério de classificação canteiro seria descartado. Então o modelo seria (Equação 3):

Tabela 2. Desvios padrão (incertezas padrão) incluindo os dois resultados - os originais e os robustos - publicados no Guia e os presentemente obtidos com descarte de valores aberrantes (outliers).

\begin{tabular}{lllc}
\hline \multicolumn{1}{c}{ Causas variação } & Originais $^{\mathrm{a}}$ & Robustos $^{\mathrm{a}}$ & Com descarte \\
\hline Entre canteiros & 556,2804 & 565,39868 & 641,22757 \\
Alfaces d. canteiros & 518,16089 & 319,04834 & 287,16458 \\
Análises (quartos, erros) & 148,18063 & 167,94308 & 232,23135 \\
Medição & $538,9325^{\text {nc }}$ & 360,5506 & 369,3168 \\
\hline
\end{tabular}

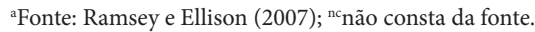

$y_{i j k}=\mu+a_{i j}+\varepsilon_{i j k}$,

onde $y_{i j k}$ é o valor observado (medido), $\mu$ a média, $a_{i j}$ o erro da alface $_{i j}, i=1,2, \ldots, 16, j=1,2, \ldots 8$, e $\varepsilon_{i j k}$ o erro de análises, $k=1,2, \ldots$ 32 , ou efeito do quarto ${ }_{k}$, dentro no alface $_{i j}$.

Mas, $a_{i j}, \mathrm{i}=1,2, \ldots, 16, \mathrm{j}=1,2, \ldots 8$ e $\varepsilon_{i j k}$ são erros que podem ser expressos por $\delta_{\mathrm{ij}}=a_{i j}+\varepsilon_{i j k}$, então o modelo se resume a $\mathrm{y}_{\mathrm{ij}}=\mu+\delta_{\mathrm{ij}}$. Então o modelo proposto pelo Guia fica descaracterizado, bem como os demais nele baseados. $\mathrm{O}$ efeito de canteiros deixa de existir, tornando-se o que é a realidade de um processo amostral, que tem, como em todo o tipo de amostra estocástica, uma única média e não há "variância de amostragem".

Se houvesse diferença significativa entre duas amostras -estocasticamente planejadas e coletadas - acabaria toda e qualquer possibilidade de existência da própria Estatística, pois não existiria o que se chama de amostra representativa, um absurdo para o Método Científico (método indutivo - dedutivo, ou hipotético - dedutivo).

\subsection{Incerteza e verificação de conformidade}

Neste item passa-se a abordar o conceito estocástico de amostra.

Envolvendo a questão da variância (incerteza) como um todo, como sugere FAO e WHO (FOOD...; WORLD.., 2008), na literatura ocorrem diferenças significativas de variâncias (precisões, incertezas) de mensurandos até entre matrizes de mesma natureza. Normalmente ocorrem variações nas variâncias dos mensurandos em função de diferenças de variedade, de clima, adubação, etc.

No entanto, é preciso conviver naturalmente com variabilidade e com riscos amostrais. Variabilidade existe, é característica da natureza e de processos, ou média não existiria. Variação em um processo provém de fontes tão naturais quanto em um fenômeno da natureza. Não há razão de espanto ou tentativa de correção de propriedades que são naturais.

A amostragem ou modelagem realizada uma só vez não é representativa do resultado de incertezas de sistemas de processamento de uma indústria de alimentos ou de produções de um campo agrícola, que são temporais. No entanto, a validade da incerteza obtida segundo a modelagem em Ramsey e Ellison (2007) no Guia não pode ser admitida além do campo ou do momento em que foi realizada num processo.

Paradoxalmente a literatura menciona a busca do intervalo em que está o valor verdadeiro. O que se deve perguntar é: qual dos valores verdadeiros? É o valor verdadeiro do mensurando na porção que está sendo medida? É o valor verdadeiro do mensurando em todo o material coletado? É o valor verdadeiro do mensurando no lote? É o valor verdadeiro do mensurando na plantação? Qual é o valor verdadeiro que se procura?

De acordo com Guia EURACHEM/CITAC de Ellison e Williams (2007), o objetivo dos atuais cálculos de incerteza é verificação de conformidade, ou seja, utilizar seu resultado para decidir se ele indica conformidade ou não de certo resultado com uma especificação. 
Então, não é a variância total (incerteza total) do processo de medição que vem sendo procurada, mas algo que extrapola os limites de medição e os limites físicos do próprio laboratório, um estimador de uma variância em que todas, e absolutamente todas as possíveis causas de variação do processo de produção agrícola ou agroindustrial, estejam representadas de alguma forma ou implícitas, e não somente o de medição.

Em outras palavras, o que vem sendo procurado é uma variância genérica que possa ser usada para representar a variação de um processo produtivo, industrial ou agrícola no decorrer do tempo e do espaço, que possa ser usada para caracterizar o processo, possibilitar verificação de conformidade e conter o valor verdadeiro da média do processo como um todo para poder rejeitar, ou não, um lote ou qualquer produção como não conforme com certa especificação.

Sem dúvida, o problema é estocástico, é inferencial, e a solução somente poderia ser estocástica. A solução está em pensar o problema como um todo, como sugere FAO e WHO (FOOD...; WORLD.., 2008), e não somente na medição dentro dos laboratórios. A solução exige representar adequadamente o processo, a produção, etc., o que só se consegue com um plano amostral especialmente preparado para o processo/população estudado(a).

No caso de processos industriais, agrícolas, etc. um ou mais planos seriam continuados até se obter convergência. Poderiam também ser usados estimadores bayesianos, ou bayesianos acoplados aos robustos, e outros.

Assim, a solução é estatística e não cabe a um laboratório de serviços de análises químicas, físico-químicas, etc. A este cabe ensaiar o método analítico, controlar condições de ensaio, realizar treinamentos, etc. e não o mapeamento/rastreamento/ pesquisa de estimadores de variância do processo produtivo, agrícola, industrial, social, natural, etc.

Para isso há os métodos de amostragem, incluindo-se aqueles normalizados, que podem ser aplicados em cada caso atendendo sua especificidade, por exemplo em: ISO 3534-2 (INTERNATIONAL..., 1993) que traz um esquema geral, na série ISO 3951-1 a 5 (INTERNATIONAL..., 2005, 2006a, b, 2007), que abrange desde os processos em batelada quanto os contínuos e outros.

A estimativa de incerteza de uma propriedade de um processo/lote/etc. é uma estimativa que uma indústria deve ter e manter sob controle estatístico (controle estatístico de qualidade, ou de processos, capabilidade) assim como um exportador agrícola ou agroindustrial ou similar, e não é elaborada/pesquisada por um laboratório de ensaios, enquanto que os laboratórios podem somente se voltar ao que se refere a seu controle interno de métodos de ensaio.

Finalmente, tal variância (do processo, lote, etc.) será usada para se traçar um intervalo de confiança do todo, do modo como se expressa FAO e WHO (FOOD...; WORLD.., 2008), a aproximadamente $95 \%$, o que é traduzido pela expressão $\times \pm 2$ segundos em casos de normalidade, onde s é o desvio padrão do todo.
Porém essa expressão $\times \pm 2$ segundos equivale a um teste de hipóteses bilateral. Os testes de hipóteses têm regras de conclusão reconhecidas como válidas e são aceitos no meio científico, de forma que a decisão na Figura 2, que rege a verificação de conformidade pode ser mantida ou substituída, com mais clareza, por este tipo de teste, ou seja, pelo teste para se verificar se o resultado de amostragem está conforme com a especificação.

A regra de conclusão existente para os testes de hipóteses bilaterais resulta nos casos (b) e (c) não rejeitarem a hipótese de conformidade. Assim, não há, estocasticamente, dúvida sobre estas duas situações e a verificação de conformidade pode ser realizada normalmente.

Quanto ao controle de qualidade intralaboratorial, as Instituições prestadoras de serviços de análises devem estar cientes de que alterações significativas ocorrem nas incertezas até entre matrizes de baixo teor de umidade e da mesma natureza (por exemplo, entre farinhas de trigo). Poderão, então, aferir o comportamento da incerteza (variância), vícios, etc. de seus métodos mediante o uso de padrões ou materiais de referência pesquisados para estes fins, ou mesmo através de resultados satisfatórios em ensaios de proficiência e similares.

\section{Conclusões}

Foram encontrados comportamentos de incertezas (variação, dispersão) em analitos importantes na área de C\&T de alimentos, como quantidade de micotoxinas, teor de sódio, e até em curvas de calibração cromatográficas em que não se mostrou possível obter diversas estimativas válidas de incerteza de medição, até mesmo por componentes. Mesmo que se tentasse usar funções que sejam identificadas em alguns casos, isto contrariaria a própria definição de incerteza, na Estatística e no VIM, e resulta que não se pode obter uma incerteza válida proveniente de um laboratório nestes casos.

A proposta do Guia de incerteza de amostragem foi discutida e se concluiu pela sua invalidade. Assim, a solução presentemente proposta para a estimação de incerteza para fins de verificação de conformidade em química de alimentos é estocástica, extrapola os limites físicos e a competência técnica e legal dos laboratórios prestadores de serviços de análises.

Para fins de decisão quanto à conformidade de produtos, devem ser usadas as conhecidas técnicas de amostragem e regras de testes de hipóteses estocásticos. Nelas o segundo e o terceiro caso do Guia EURACHEM/CITAC/Nordtest de Ellison e Willians (2007) de verificação de conformidade implicam não rejeição da conformidade.

Para fins de validação/controle laboratorial de métodos analíticos, devem ser usados ensaios de proficiência, padrões, comparações interlaboratoriais, etc.

Enfim, é necessário lembrar o comentário de Horwitz e Albert (1997), segundo o qual os cálculos de incerteza recomendados para medidas físicas não são transferidos imediatamente para a química. Ou seja, há complicações nas medições químicas que resultam que as abordagens de incerteza por componentes ou conforme o Guia de Ramsey e Ellison (2007) não são satisfatórias e trazem riscos se utilizadas por laboratórios de análises. 


\section{Referências bibliográficas}

ASSOCIAÇÃO BRASILEIRA DE NORMAS TÉCNICAS; I N T E R N A T I O N A L O R G A N I Z A T I O N F O R STANDARDIZATION; INTERNATIONALELETROTECHNICAL COMMISSION. ABNT / ISO / IEC Guia 43-1: Ensaios de proficiência por comparações interlaboratoriais - Parte 1: Desenvolvimento e operação de programas de ensaios de proficiência. Rio de Janeiro, 1999.

ASSOCIAÇÃO BRASILEIRA DE NORMAS TÉCNICAS; INTERNATIONAL ORGANIZATIONFORSTANDARDIZATION; INTERNATIONAL ELETROTECHNICAL COMISSION. NBR / ISO / IEC 17025: Requisitos gerais para competência de laboratórios de ensaio e calibração. Rio de Janeiro, 2001.

ELLISON, S. L. R.; ROESSLEIN, M.; WILLIAMS, A. EURACHEM / CITAC Guide: Quantifying uncertainty in analytical measurement. 2. ed. Teddington: EURACHEM, 2000. 122 p.

ELLISON, S. L. R.; WILLIAMS, A. EURACHEM / CITAC Guide: Use of uncertainty information in compliance assessment. Teddington: EURACHEM, 2007. 15 p.

EUROPEAN COOPERATION FOR ACCREDITATION. EA-4/02: Expression of uncertainty of measurement in calibration. Paris, 1999. $79 \mathrm{p}$.

FOOD AND AGRICULTURE ORGANIZATION - FAO; WORLD HEALTH ORGANIZATION - WHO. Codex Alimentarius Commission. Codex Committee on Methods of Analysis and Sampling. Guidance on measurement uncertainty and uncertainty of sampling. Budapeste, 2008. Agenda item 7. CX/MAS 08/29/9. Disponível em: <ftp://ftp.fao.org/codex/ccmas29/ma29_09e.pdf >. Acesso em: 27 jun. 2008.

GONÇALVES, E. B. et al. Busca de estimativas de incerteza em medições analíticas em laboratórios de alimentos. Brazilian Journal of Food Technology, v. 9, n. 4, p. 237-247, 2006.

HOGG, R. V.; CRAIG, A. T. Introduction to mathematical statistics. 4. ed. Londres: Collier Macmillan, 1978. 438 p.

HORWITZ, W.; ALBERT, R. The concept of uncertainty as applied to chemical measurements. The Analyst, 1997, vol. 122, n. 6, p. 615-617.

INSTITUTO NACIONAL DE METROLOGIA, NORMALIZAÇÃO E QUALIDADE INDUSTRIAL. DOQ-CGCRE-008: Orientações sobre validação de métodos de ensaios químicos. rev. 1. Rio de Janeiro: INMETRO, 2003. 36 p.

INSTITUTO NACIONAL DE METROLOGIA, NORMALIZAÇÃO E QUALIDADE INDUSTRIAL. DOQ-CGCRE-008: Orientação sobre validação de métodos de ensaios químicos. rev. 2. Rio de Janeiro: INMETRO, 2007. 25 p.

INSTITUTO NACIONAL DE METROLOGIA, NORMALIZAÇÃO E QUALIDADE INDUSTRIAL; CONFEDERAÇÃO NACIONAL DA INDÚSTRIA; SERVIÇO NACIONAL DE APRENDIZAGEM INDUSTRIAL. Vocabulário internacional de termos fundamentais e gerais de metrologia. Brasília: INMETRO / CNI / SENAI, 2000. $75 \mathrm{p}$.

INTERNATIONAL ORGANIZATION FOR STANDARDIZATION. ISO 3534-2: Statistics - Vocabulary and symbols - Part 2: Statistical quality control. Genéve, 1993.

INTERNATIONAL ORGANIZATION FOR STANDARDIZATION. ISO 5725-1: Accuracy (trueness and precision) of measurement methods and results; Part 1: General principles and definitions. Genève, 1994a.
I N T E R N A T I O N A L O R G A N I Z A T I O N F O R STANDARDIZATION. ISO 5725-2: Accuracy (trueness and precision) of measurement methods and results; Part 2: Basic method for the determination of repeatability and reproducibility of a standard measurement method. Genève, 1994b.

INTERNATIONAL ORGANIZATION FOR STANDARDIZATION. ISO / TS 21748: Guidance for the use of repeatability, reproducibility and trueness estimates in measurement uncertainty estimation. Genéve, 2004. 30 p.

INTERNATIONAL ORGANIZATION FOR STANDARDIZATION. ISO 3951-1: Sampling procedures for inspection by variables - Part 1: Specification for single sampling plans indexed by acceptance quality limit (AQL) for lot-by-lot inspection for a single quality characteristic and a single AQ. Genéve, 2005.

INTERNATIONAL ORGANIZATION FOR STANDARDIZATION. ISO 3951-2: Sampling procedures for inspection by variables Part 2: General specification for single sampling plans indexed by acceptance quality limit (AQL) for lot-by-lot inspection of independent quality characteristics. Genéve, 2006a.

INTERNATIONAL ORGANIZATION FOR STANDARDIZATION. ISO 3951-5: Sampling procedures for inspection by variables - Part 5: Sequential sampling plans indexed by acceptance quality limit (AQL) for inspection by variables (known standard deviation). Genéve, 2006b.

INTERNATIONAL ORGANIZATION FOR STANDARDIZATION. ISO 3951-3: Sampling procedures for inspection by variables - Part 3: Double sampling schemes indexed by acceptance quality limit (AQL) for lot-by-lot inspection. Genéve, 2007.

INTERNATIONAL ORGANIZATION FOR STANDARDIZATION; INTERNATIONAL ELETROTECHNICAL COMISSION. ISO / IEC 17025: General requirement for the competence of testing and calibration laboratories. Genéve, 2005.

MATSUSHITA, R. Y. O que é a estatística? Disponível em: <http:// www.unb.br/ie/est/>. Acesso em: 21 jul. 2008

RAMSEY, M. H.; ELLISON, S. L. R. EURACHEM / EUROLAB / CITAC / Nordtest / AMC Guide: Measurement uncertainty arising from sampling, a guide to methods and approaches. Teddington: EURACHEM, 2007. 102 p.

SCHEFFÉ, H. A statistical theory of calibration. Annals of Statistics, v. 1, n. 1, p. 1-37, 1973.

TAYLOR, B. N.; KUYATT, C. E. NIST Technical note 1297, Guidelines for evaluating and expressing the uncertainty of NIST measurement results. Gaithersburg: NIST, 1993.

THOMPSON, N.; STEPHEN, L. R.; WOOD, R. Harmonized guidelines for the single laboratory validation of methods of analysis, IUPAC Technical Report. Pure and Applied Chemistry, v. 74, n. 5, p. 835-855, 2002.

UNITED KINGDOM ACCREDITATION SERVICE. The expression of uncertainty in testing. Feltham: UKAS, 2000. 13 p.

WORKING PARTY ON CHEMICAL CONTAMINANTS IN FOODS 2003. Annex to WPCC / 2003 / 9; Report of the standing committee on the food chain and animal, Health on the relationship between analytical results, The measurement of uncertainty recovery, Factors and the provisions in EU food and feed legislation. Disponível em: <http://www.foodstandards.gov.uk/multimedia/ pdfs/wpcc20039annex.pdf>. Acesso em: 20 maio 2004. 